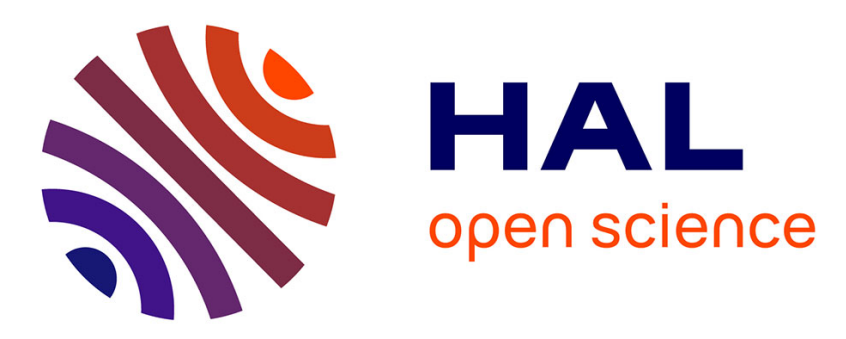

\title{
High-order h-adaptive discontinuous Galerkin methods for ocean modelling
}

Paul-Emile Bernard, Nicolas Chevaugeon, Vincent Legat, Éric Deleersnijder, Jean-François Remacle

\section{- To cite this version:}

Paul-Emile Bernard, Nicolas Chevaugeon, Vincent Legat, Éric Deleersnijder, Jean-François Remacle. High-order h-adaptive discontinuous Galerkin methods for ocean modelling. Ocean Dynamics, 2007, 57 (2), pp.109-121. 10.1007/s10236-006-0093-y · hal-01006928

\section{HAL Id: hal-01006928 \\ https://hal.science/hal-01006928}

Submitted on 22 Jan 2018

HAL is a multi-disciplinary open access archive for the deposit and dissemination of scientific research documents, whether they are published or not. The documents may come from teaching and research institutions in France or abroad, or from public or private research centers.
L'archive ouverte pluridisciplinaire HAL, est destinée au dépôt et à la diffusion de documents scientifiques de niveau recherche, publiés ou non, émanant des établissements d'enseignement et de recherche français ou étrangers, des laboratoires publics ou privés. 


\title{
High-order h-adaptive discontinuous Galerkin methods for ocean modelling
}

\author{
Paul-Emile Bernard • Nicolas Chevaugeon • \\ Vincent Legat - Eric Deleersnijder • \\ Jean-François Remacle
}

\begin{abstract}
In this paper, we present an $h$-adaptive discontinuous Galerkin formulation of the shallow water equations. For a discontinuous Galerkin scheme using polynomials up to order $p$, the spatial error of discretization of the method can be shown to be of the order of $h^{p+1}$, where $h$ is the mesh spacing. It can be shown by rigorous error analysis that the discontinuous Galerkin method discretization error can be related to the amplitude of the inter-element jumps. Therefore, we use the information contained in jumps to build error metrics and size field. Results are presented for ocean modelling problems. A first experiment shows that the theoretical convergence rate is reached with the discontinuous Galerkin high-order $h$-adaptive method applied to the Stommel wind-driven gyre. A second experiment shows the propagation of an anticyclonic eddy in the Gulf of Mexico.
\end{abstract}

Keywords Shallow water equations $\cdot H$-adaptivity Discontinuous Galerkin · A posteriori error estimation

P.-E. Bernard . V. Legat . E. Deleersnijder . J.-F.

Remacle Center for Systems Engineering and

Applied Mechanics

(CESAME), Université Catholique de Louvain,

Avenue Georges Lemaître 4,

1348 Louvain-la-Neuve, Belgium

e-mail: bernard@mema.ucl.ac.be

E. Deleersnijder

Institut d'Astronomie et de Géophysique G.

Lemaître, Université Catholique de Louvain,

Chemin du cyclotron 2,

1348 Louvain-la-Neuve, Belgium

N. Chevaugeon · J.-F. Remacle

Département d'Architecture, d'Urbanisme de Génie Civil

et Environnemental, Université Catholique de Louvain,

Place du Levant 1 ,

1348 Louvain-la-Neuve, Belgium

\section{Introduction}

The discontinuous Galerkin (DG) method has become a very attractive method especially for advection-dominated problems (e.g. Cockburn et al. 2000; Adjerid et al. 2002; Bassi and Rebay 1997). The main advantage is its flexibility in terms of mesh and shape functions. Moreover, the compactness of the stencil is maintained for high order efficient parallel implementation. Recent advances coming from the integration-free version of the formulation (e.g. Lockard and Atkins 1999; Atkins and Shu 1998) allow for an enhancement of the computational efficiency of the DG method. The quadrature free implementation is especially useful at high polynomials orders.

In our work, we aim to develop a global ocean circulation model where the geometry is complex enough to justify the shift from traditional structured grids models to unstructured meshes (e.g. Hanert et al. 2004; Pietrzak et al. 2005). In ocean modelling, important dynamics features like meso-scale eddies have to be followed in time and solved accurately. The ocean exhibits many different length scales in time and space, with very unsteady behaviour and almost discontinuous fields. The meso-scale processes contain a huge part of the ocean energy and have to be captured. Dynamic mesh adaptation strategies following those structures represent a great potential in the field of ocean modelling (e.g. Behrens 1998; Heinze and Hense 2002; Nair et al. 2005; Giraldo et al. 2002). In this framework of a new unstructured ocean model, we believe that the DG method is a good candidate because it provides a simple and efficient error estimator for any order $p$, which means a simple and efficient way to deal with mesh adaptivity. Moreover, the DG method ensures local conservation, which may be a critical issue in ocean modelling, and is particularly efficient for advection-dominated problems.

Recent applications (e.g. Baker 1997; Speares and Berzins 1997; George et al. 2002; Chevaugeon et al. 2005c) show that transient mesh adaptation technologies are mature enough to tackle difficult problems. The computational overhead of modifying the mesh is negligible compared to the overall gain in computation time and accuracy. 
Starting from a fast implementation of the DG method, originally developed to solve wave propagation problems (e.g. Chevaugeon et al. 2005b), we first discuss the implementation of the shallow water equations, in particular the choice of an appropriate Riemann solver. After a brief description of the mesh-adaptation package, MeshAdapt developed at $\operatorname{SCOREC}^{1}$ (e.g. Remacle et al. 2005; Li 2003), we detail the mesh adaptation strategy based on the error estimation for the DG method. We then provide some preliminary validations of the method by solving the classical Stommel model, before turning to an idealized simulation of an anticyclonic baroclinic eddy in the Gulf of Mexico.

\section{DG method for shallow water equations}

It is only recently that the DG method has been applied to the shallow water equations (e.g. Schwanenberg and Kongeter 2000; Dawson and Proft 2002, 2004; Nair et al. 2005; Giraldo et al. 2002). These equations have been used for many years for solving a variety of problems, such as atmospheric, oceanic, dam breaking (e.g. Soares Frazão and Zech 2002b; Remacle et al. 2006) or river flow problems (e.g. Vreugdenhill 1994).

\subsection{Shallow water equations}

The shallow water equations describe the flow of a thin layer of incompressible fluid, with no stratification, under the influence of a gravitational force. This model is based on the assumption that the vertical dimension is very small compared to the horizontal one. A vertical integration over the depth of the fluid layer $H(x, t)=h(x)+\eta(x, t)$ (where $h$ is the unperturbed height of the water column and $\eta$ the surface elevation measured from a reference height; Fig. 1) is then performed on the 3D Navier-Stokes equations. The bottom and the surface of the ocean are impermeable, which yields the two boundary conditions required for integration.

The two-dimensional, conservative form of the shallow water equations is then obtained, not in terms of pressure and velocity, but in terms of water depth and mean velocity:

$$
\begin{aligned}
& \frac{\partial H}{\partial t}+\nabla \cdot(H \mathbf{v})=0 \\
& \frac{\partial H \mathbf{v}}{\partial t}+\nabla \cdot(H \mathbf{v v})+g H \nabla \eta+f \mathbf{e}_{z} \times H \mathbf{v}=\frac{\tau^{\mathrm{s}}-\boldsymbol{\tau}^{\mathrm{b}}}{\rho}
\end{aligned}
$$

where $t$ is time, $f$ is the Coriolis parameter, $\mathbf{v}$ is the depthaveraged horizontal velocity, $g$ is the gravitational acceleration, $\tau^{s}$ and $\tau^{b}$ denote the surface and bottom stresses, respectively.

\footnotetext{
${ }^{1}$ SCOREC, Scientific Computation Research Center, Rensselaer Polytechnic Institute, Troy, NY, USA.
}

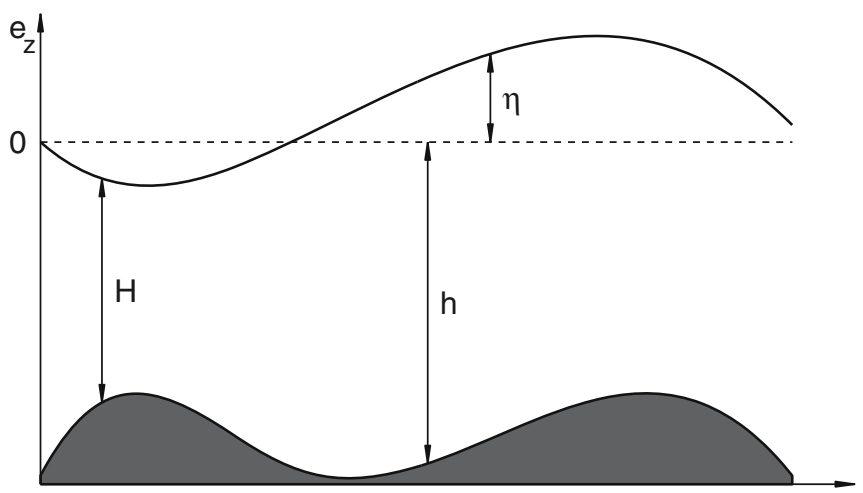

Fig. 1 Shallow water notations for water depth $H$ with a timeindependent bathymetry $h$. Notice that the relative elevation $\eta$ is usually several orders of magnitude smaller than the unperturbed depth

The main parameters are

1. The Rossby number: $R o=\frac{U}{L f}$, with $U$ a characteristic velocity and $L$ a characteristic length. The Rossby number is the ratio between the Earth period and the flow period. It represents the relative importance of the Coriolis effect.

2. The Froude number: $F r=\frac{U}{c}$, with $c$ the speed of the gravity waves. The Froude number represents the ratio between the flow velocity and the gravity waves velocity. It is similar to the Mach number in compressible fluid dynamics problems. The flow is said to be critical when the Froude number reaches $F r=1$.

The free surface allows propagation of gravity waves at speed $c=\sqrt{g H}$ (those are equivalent to sound waves in Euler equations). In the case of an ocean modelling problem, the speed of gravity waves is typically 100 to 1,000 times faster than the speed of the fluid itself.

\subsection{DG method applied to shallow water equations}

We consider a closed two dimensional domain $\Omega$. Its boundary $\partial \Omega$ has a normal $n$ defined everywhere. We seek to determine the vector of unknowns $\mathbf{U}(\Omega, t)$ as the solution of a system of conservation laws:

$\frac{\partial \mathbf{U}}{\partial t}+\nabla \cdot \mathbf{F}(\mathbf{U})=\mathbf{S}$,

where $\mathbf{F}$ is the flux matrix and $\mathbf{S}$ is the vector containing the source terms.

We multiply Eq. 3 by a test function $\mathbf{w}$ and integrate on the domain $\Omega$ to obtain this classical weak formulation:

$\left\langle\partial_{t} \mathbf{U}, \mathbf{w}\right\rangle_{\Omega}+\langle\nabla \cdot \mathbf{F}(\mathbf{U}), \mathbf{w}\rangle_{\Omega}=\langle\mathbf{S}, \mathbf{w}\rangle_{\Omega}$,

with the scalar product: $\langle a, b\rangle_{v}=\int_{v} a b d v$. 
The computational domain is divided into a set of elements $\Omega_{e}$ called a mesh. In the case of a DG method, we approximate the unknown fields using piecewise discontinuous polynomial approximations: in element $\Omega_{e}$, the fields $\mathbf{U}$ are approximated using $p$-order polynomials in each element with no inter element continuity requirements. The total number of degrees of freedom for a fully triangular mesh of $N$ elements is therefore equal to $N \times[(p+1)(p+2) / 2] \times m$, with $m$ the number of unknown fields, i.e. three in the shallow water case. Because all approximations are disconnected, the weak form Eq. 4 can be written in each element. After having integrated the divergence of fluxes by parts in Eq. 4, we obtain

$$
\begin{aligned}
\left\langle\partial_{t} \mathbf{U}, \mathbf{w}\right\rangle_{\Omega e} & -\langle\mathbf{F}(\mathbf{U}), \nabla \mathbf{w}\rangle_{\Omega e}+\langle\mathbf{F}(\mathbf{U}) \cdot \boldsymbol{n}, \mathbf{w}\rangle_{\partial \Omega e} \\
& =\langle\mathbf{S}, \mathbf{w}\rangle_{\Omega e} .
\end{aligned}
$$

A numerical flux function has to be supplied to the formulation because unknowns $\mathbf{U}$ are multiply valued at element interfaces $\partial \Omega_{e}$.

Two neighbouring elements in continuous finite element method share common nodes that ensure the continuity of the finite element approximation. With the DG method, fields are discontinuous through element edges. Jumps at element interfaces have to be controlled by a numerical flux function. In this paper, we consider triangular meshes exclusively. The boundary $\partial \Omega_{e}$ of a triangle $\Omega_{e}$ is composed of three edges $\partial \Omega_{e_{k}}, k=1, \ldots, 3$. The flux function is computed on those three edges using a combination of the fields on both sides of the edge, i.e. using the unknown fields $\mathbf{U}$ inside element $\Omega_{e}$ and using the unknown fields $\mathbf{U}^{k}$ in the neighbouring triangle across edge $\partial \Omega_{e_{k}}$ We have:

$$
\langle\mathbf{F}(\mathbf{U}) \cdot \boldsymbol{n}, \mathbf{w}\rangle_{\partial \Omega_{e}}=\sum_{k=1}^{3}\left\langle\mathbf{F}_{n}\left(\mathbf{U}, \mathbf{U}^{k}\right), \mathbf{w}\right\rangle_{\partial \Omega_{e_{k}}} .
$$

The centered DG scheme uses the average of fluxes as the flux function:

$\mathbf{F}_{n}\left(\mathbf{U}, \mathbf{U}^{k}\right)=\frac{1}{2}\left(\mathbf{F}(\mathbf{U})+\mathbf{F}\left(\mathbf{U}^{k}\right)\right) \cdot \boldsymbol{n}$.

Though producing no spatial dissipation, the use of a centered scheme may cause advective unstabilities when the discretization is unable to resolve a certain range of wave numbers (e.g. Chevaugeon et al. 2005b). Riemann solvers are the extension of upwind schemes to non-linear systems of conservation laws.

The idea of the Riemann solver consists in upwinding the characteristics variables. The projection of Eq. 3 without source terms on the normal direction $\mathbf{n}$ is written as:

$\frac{\partial \mathbf{U}}{\partial t}+\frac{\partial \mathbf{F}_{\mathbf{n}}(\mathbf{U})}{\partial n}=\frac{\partial \mathbf{U}}{\partial t}+\mathbf{A}_{\mathbf{n}} \frac{\partial \mathbf{U}}{\partial n}=0$,

where $\mathbf{A}_{\mathbf{n}}=\frac{\partial \mathbf{F}_{\mathbf{n}}}{\partial \mathbf{U}}$ is the jacobian matrix of the flux vector in the normal direction $\mathbf{F}_{\mathbf{n}}$. This jacobian matrix can be written as $\mathbf{A}_{\mathbf{n}}=\mathbf{R} \mathbf{\Lambda} \mathbf{R}^{-1}$ with matrices of eigenvectors $\mathbf{R}$ and eigenvalues $\boldsymbol{\Lambda}$. We can then derive the following onedimensional transport equation:

$\frac{\partial \mathbf{U}^{*}}{\partial t}+\Lambda \frac{\partial \mathbf{U}^{*}}{\partial x}=0$

The characteristics variables $\mathbf{U}^{*}=\mathbf{R}^{-1} \mathbf{U}$, the Riemann invariants, are convected along the normal direction to the edge of the element. The transport velocities are the eigenvalues of the problem, which are used to choose the appropriate values on the edge. More precisely, upwinding can be applied on the characteristics variables convected across the edge. Note that the source terms $\mathbf{S}$ are not taken into account in Eq. 6 because they have no influence on the Riemann invariants or on the sign of the eigenvalues neither. Note also that no diffusion term was considered because the Riemann solver only deals with advection. This diffusion term can be solved in the usual way with an integration by part and a centered scheme to define the interface values. It has been shown that this Riemann solver introduces the minimum numerical dissipation required to stabilize the numerical scheme in the presence of transport terms.

The shallow water equations lead to the following expressions for $\mathbf{U}$ and $\mathbf{F}$ :

$\mathbf{U}=\left[\begin{array}{c}H \\ H u \\ H v\end{array}\right], \quad \mathbf{F}(\mathbf{U})=\left[\begin{array}{cc}H u & H v \\ H u u+g \frac{H^{2}}{2} & H u v \\ H v u & H v v+g \frac{H^{2}}{2}\end{array}\right]$.

The eigenvalues matrix read:

$\mathbf{\Lambda}=\left[\begin{array}{ccc}\mathbf{v} \cdot \mathbf{n}+c & 0 & 0 \\ 0 & \mathbf{v} \cdot \mathbf{n}-c & 0 \\ 0 & 0 & \mathbf{v} \cdot \mathbf{n}\end{array}\right]$

where $c=\sqrt{g h}$ is the gravity waves velocity.

To keep the same general formulation in Eq. 3, the transport terms require the use of the conservative formulation. Advection terms are thus expressed as a divergence $\nabla \cdot(H \mathbf{v v})$. This conservative form is non-linear, even without transport terms, because of the presence of the elevation term $\nabla\left(\frac{H^{2}}{2}\right)$. But the transport terms lead to a complex and computationally prohibitive exact Riemann solver. Approximate Riemann solvers are proved to 
produce more numerical dissipation than the exact solver, but numerical experience suggests that this choice does not have a significant impact on the accuracy of the solution, especially when polynomial degree increases. The conservative formulation can thus be solved with, for example, a Roe solver, which consists of the exact solution to a linearized Riemann problem, and is consistent with the discrete entropy condition (e.g. Roe 1981). The basic idea consists in considering that over a small time step, the characteristics curves propagating information can be replaced by straight lines. This approximation leads to consider as constant the eigenvalues and eigenvectors matrices $\Lambda$ and $R$.

The Roe numerical flux for shallow water equations (e.g. Remacle et al. 2006; Soares Frazão and Zech 2002a) can be written as:

$$
\begin{aligned}
\mathbf{F}_{n}\left(\mathbf{U}, \mathbf{U}^{k}\right)= & \frac{1}{2}\left[\mathbf{F}(\mathbf{U})+\mathbf{F}\left(\mathbf{U}^{k}\right)\right] \cdot \boldsymbol{n}+ \\
& \frac{1}{2} \operatorname{Fr}\left[\mathbf{F}(\mathbf{U})-\mathbf{F}\left(\mathbf{U}^{k}\right)\right] \cdot \boldsymbol{n}+ \\
& \frac{1}{2} c_{A}\left(1-\mathbf{F} r^{2}\right)\left[\mathbf{U}-\mathbf{U}^{k}\right] .
\end{aligned}
$$

The first term corresponds to the centered flux, the others are dissipation terms. The average Froude number $F r$ is defined as:

$F r=\frac{\boldsymbol{v}_{\boldsymbol{A}} \cdot \boldsymbol{n}}{c_{A}}$,

with $\boldsymbol{v}_{A}$ a Roe-averaged velocity and $c_{A}$ a Roe-averaged wave speed that are computed as

$$
\begin{aligned}
& u_{A}=\frac{u \sqrt{h}+u^{k} \sqrt{h^{k}}}{\sqrt{h}+\sqrt{h^{k}}}, \\
& v_{A}=\frac{v \sqrt{h}+v^{k} \sqrt{h^{k}}}{\sqrt{h}+\sqrt{h^{k}}}, \\
& c_{A}=\sqrt{g \frac{h+h^{k}}{2}} .
\end{aligned}
$$

\section{Mesh-adaptation}

Efforts on the development of mesh adaptation techniques have been underway for more than 20 years and have provided a number of important theoretical and practical results (e.g. Remacle et al. 2005; Baker 1997; Speares and Berzins 1997; George et al. 2002).

It is only recently that mesh adaptation has been applied to transient flow problems and in particular to ocean applications (e.g. Pain et al. 2005). It has been shown that DG techniques allow to control the quality of a solution transfer between two consecutive adaptive meshes (e.g.
Remacle et al. 2005). It is indeed possible to adapt the mesh very often and project solutions without alteration.

We have recently developed mesh adaptation algorithms that allow to locally modify a given $2 \mathrm{D}$ or $3 \mathrm{D}$ mesh to make it conform to a given size field (e.g. Remacle et al. 2005; Li 2003; Li et al. 2004). The MeshAdapt software package performs local mesh modifications, essentially edge swaps, edge collapses and edge splits. Note that we use here only a small set of the package capabilities; MeshAdapt is able to perform $3 D$ anisotropic mesh adaptation in parallel. It is obvious that an anisotropic mesh is the optimal choice, especially where the flow is itself anisotropic (e.g. Remacle et al. 2005). But in this framework of a first experiment in coupling ocean modelling and mesh adaptation, only isotropic meshes were considered to simplify the mesh metric definition.

\subsection{Description of the MeshAdapt package}

A mesh metric field is a smooth positive function $\mathcal{M}(x, y)$ defined over the domain $\Omega$. The length of a mesh edge $e$ is computed as $l_{e}=\int_{e} \sqrt{\mathcal{M}(x, y)} d l$. The aim of the mesh adaptation procedure is to modify an existing mesh to make it a unit mesh, i.e. a mesh for which every edge is of size $l_{e}=1$. At one given time step, a metric field is computed at every node of the mesh using the results of an a posteriori error estimation procedure. The mesh adaptation algorithm then modifies locally the present mesh by: (1) splitting all the long edges and (2) collapsing all the short edges.

Edge swaps are also performed to optimize the quality of the resulting mesh. The mesh adaptation procedure is applied iteratively until a convergence criterion is satisfied. The different local mesh modifications used here are depicted in Fig. 2.

Typically, the algorithm stops when every edge of the domain has a dimensionless size in the interval $l_{e} \epsilon$ $[0.5 ; 1.4]$. A long edge is an edge such that $l_{e}>1.4$ and a short edge is an edge of size $l_{e}<0.5$. Using this interval for short and long edges ensures that the two new edges created by a bisection will not be short edges. Oscillations between refinements and coarsenings are therefore prevented. More details about this mesh adaptation procedure can be found in previous papers (e.g. Remacle et al. 2005; Li 2003; Li et al. 2004).

As an example, we see on Fig. 3 two mesh size fields together with the respective adaptive meshes. Those results come out of a simulation that will be described below in more details. Both plots on top of Fig. 3 were computed at an early stage of the simulation while the bottom plots are the result of about 100 adaptations.

\subsection{Error estimation}

Here, we only consider the spatial error of discretization. Note that it has been shown (e.g. Chevaugeon et al. 2005a) 
Fig. 2 Local mesh modifications. Edge split (top), edge collapse (middle) and edge swap (bottom). The zone depicted in bold represents the cavity that is modified by the local mesh modification
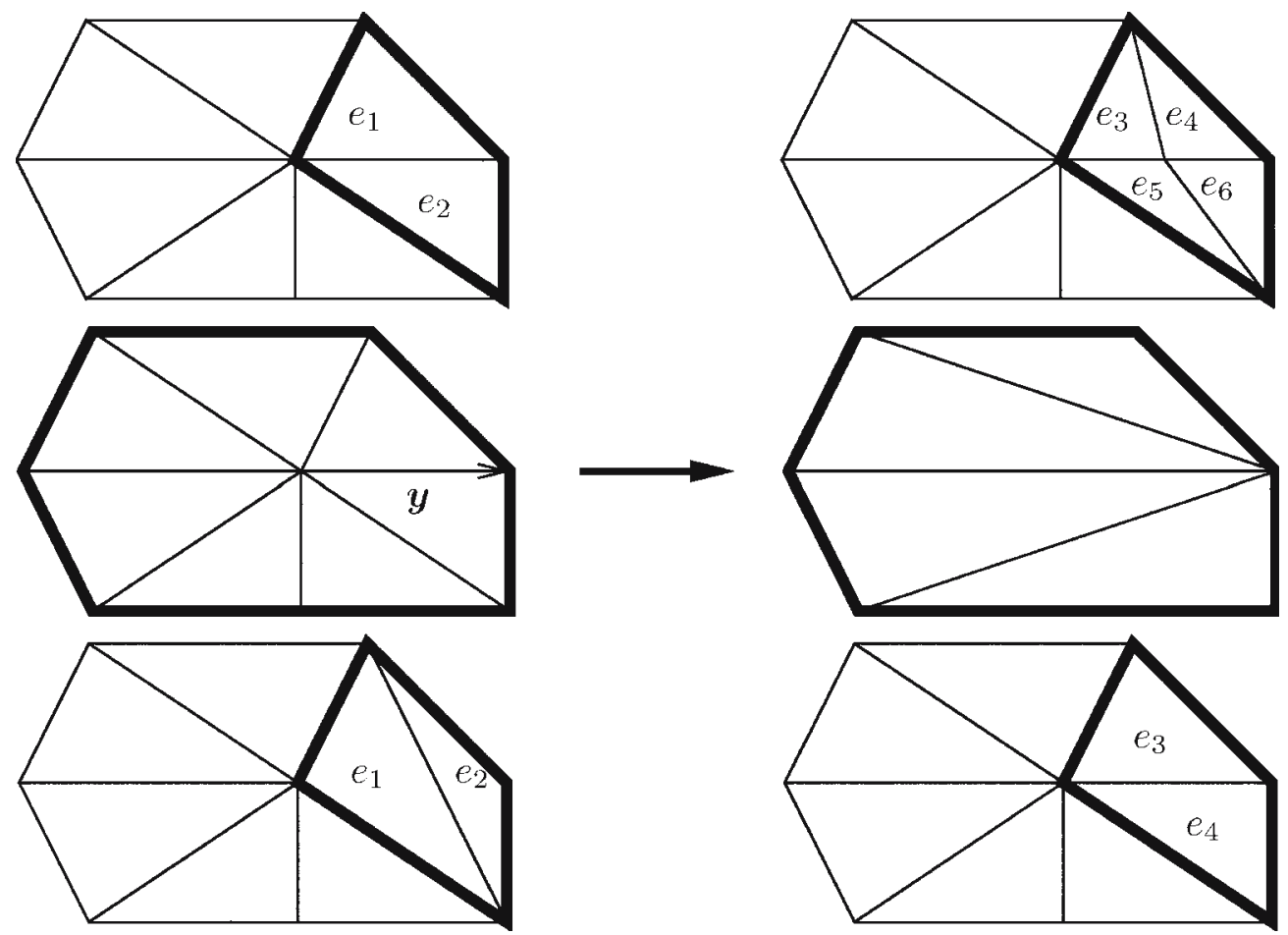

that, using an explicit Runge-Kutta time stepping of order $p+1$ in time together with a DG method of order $p$ in space, the spatial error is at least one order of magnitude higher than the error in time.
The approximated fields in a DG method are discontinuous at inter-element boundaries. It has been shown (e.g. Marchandise et al. 2005) that the inter-element jumps of the solution are converging at the same rate as the discretiza-
Fig. 3 Mesh size fields and adaptive meshes obtained at different time steps, for the propagation of a typical anticyclonic eddy in the Gulf of Mexico (cf. section 4.2). The mesh on top includes 14,545 triangles while the one on bottom includes 9,618 triangles
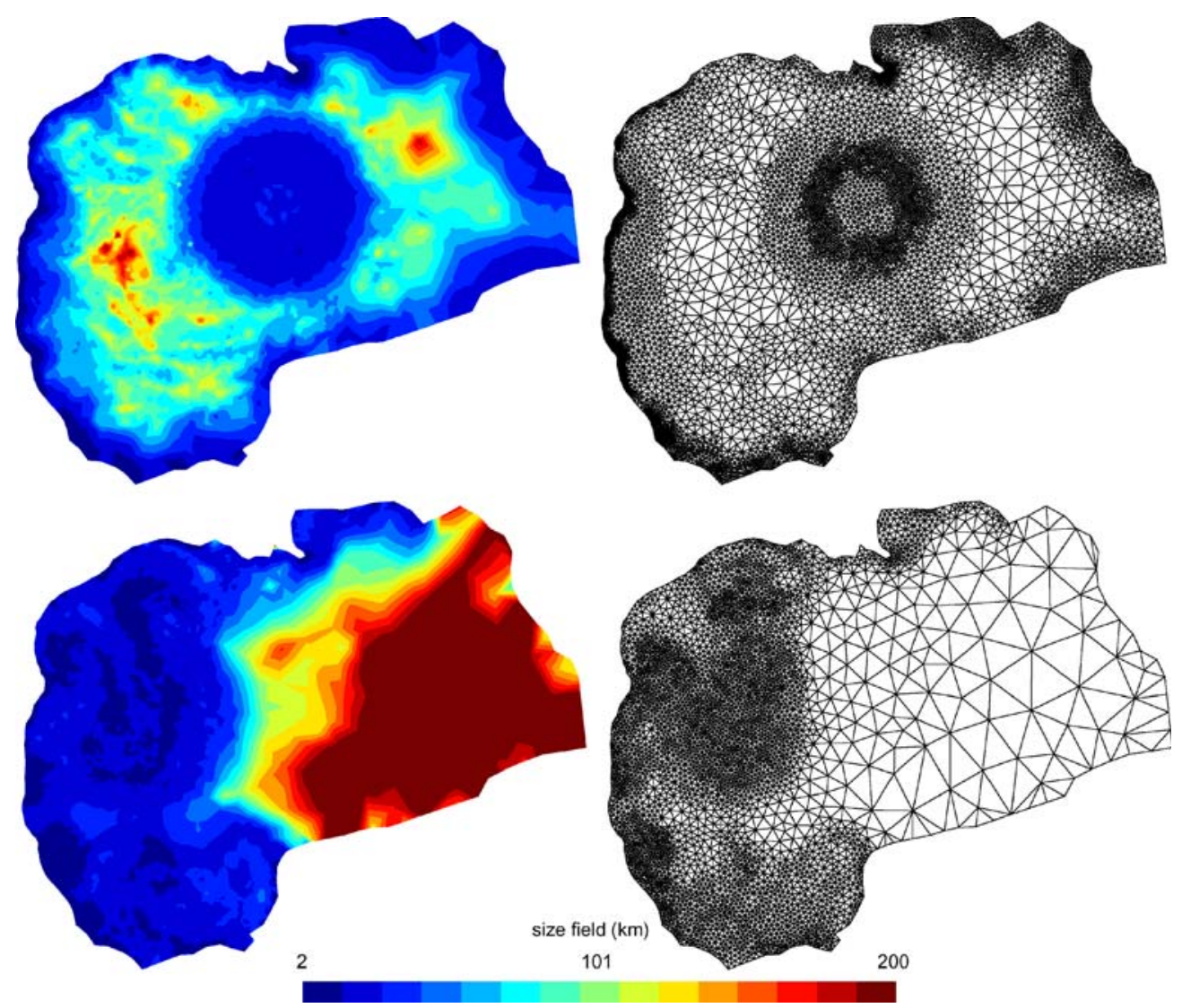
Fig. 4 An element with its three surrounding neighbors

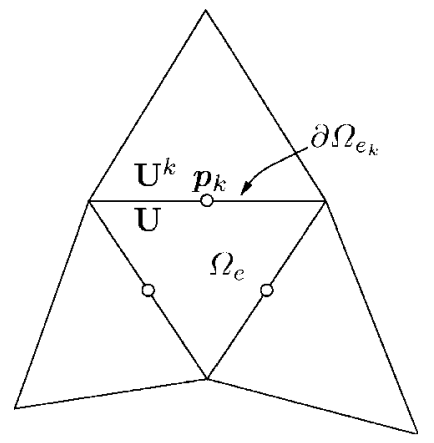

tion error. If we consider the situation depicted at Fig. 4, the jump at one point $p_{k}$ of edge $\partial \Omega_{e_{k}}$ converges at the same rate as the DG error:

$$
\left[\mathbf{U}-\mathbf{U}^{k}\right]\left(p_{k}\right)=\mathcal{O}\left(h^{p+1}\right),
$$

where $h$ is the local mesh size. Notice that $h$ always denotes the local mesh size in the following section.

It has been shown that the DG solution was superconvergent at downwind faces (e.g. Marchandise et al. 2005). This means that, on $\partial \Omega_{e_{k}}$, either $\mathbf{U}$ or $\mathbf{U}^{k}$ is a good approximation (at order $h^{2 p+1}$ ) of the exact solution. Here, we choose the average value to be the approximation of the exact solution $\mathbf{U}_{e x}$ :

$\mathbf{U}_{e x}\left(\boldsymbol{p}_{k}\right) \simeq \frac{1}{2}\left[\mathbf{U}+\mathbf{U}^{k}\right]\left(\boldsymbol{p}_{k}\right)$.

We have then:

$\mathbf{E}\left(\boldsymbol{p}_{k}\right)=\left[\mathbf{U}-\mathbf{U}_{e x}\right]\left(\boldsymbol{p}_{k}\right) \simeq \frac{1}{2}\left[\mathbf{U}-\mathbf{U}^{k}\right]\left(\boldsymbol{p}_{k}\right)$.

The jumps are therefore a good image of the discretization error and can be used as an error indicator. Here, we show that, using an appropriate measure of the jumps, we are able to use the jumps as an error estimator. We will compute local and global effectivity indices and show that they are optimal. We consider an average error along each edge.

$$
\begin{aligned}
\mathbf{e}_{\partial \Omega_{e_{k}}}^{2} & =\frac{1}{\left|\partial \Omega_{e_{k}}\right|} \int_{\partial \Omega_{e_{k}}} \mathbf{E}^{2} d s \simeq \frac{1}{4} \frac{1}{\left|\partial \Omega_{e_{k}}\right|} \int_{\partial \Omega_{e_{k}}}\left[\mathbf{U}-\mathbf{U}^{k}\right]^{2} d s, \\
\mathbf{e}_{\partial \Omega_{e_{k}}} & =\mathcal{O}\left(h^{p+1}\right) .
\end{aligned}
$$

For each element $\Omega_{e}$, we compute an a posteriori error estimator by the following rule:

$\mathbf{e}_{\Omega_{e}}^{2}=\frac{1}{3}\left|\Omega_{e}\right| \sum_{k=1}^{3} \mathbf{e}_{\partial \Omega_{e_{k}}}^{2}$, where $\left|\Omega_{e}\right|$ is the area of $\Omega_{e}$. Note that the three mid-edge points of the triangle form a Gauss quadrature rule. Clearly, using this simple approach, the error is constant in one element and the resulting size field is still constant in each high order triangle. More complex rules can be computed for higher order polynomial approximations. The total error is calculated as the sum of all elementary errors

$\mathbf{e}^{2}=\int_{\Omega} \mathbf{E}^{2} d v=\sum_{e} \mathbf{e}_{\Omega_{e}}^{2}$.

The relative error is defined as:

$\epsilon^{2}=\frac{\int_{\Omega} \mathbf{E}^{2} d v}{2 \int_{\Omega} \mathbf{U}^{2} d v}=\frac{\mathbf{e}^{2}}{2\|\mathbf{U}\|_{L^{2}}^{2}}=\mathcal{O}\left(h^{p+1}\right)$

implying that it is smaller than 1 . We define the local relative error as:

$\epsilon_{\Omega_{e}}^{2}=\frac{\mathbf{e}_{\Omega_{e}}^{2}}{2\|\mathbf{U}\|_{L^{2}}^{2}}$, with $\sum_{e} \epsilon_{\Omega_{e}}^{2}=\epsilon^{2}$

Our aim could be either to control the discretization error with a minimum number of elements or to control the number of elements in the optimal mesh while minimizing the discretization error.

Let us consider $\Omega_{e}$ an element of the initial mesh for which we have computed an error of $\epsilon_{\Omega_{e}}$. We know that, if $h_{e}$ is the size of $\Omega_{e}$ (its circumscribed radius for example) and if $h_{e}^{*}$ is the size of the elements of the optimal mesh on the region covered by $\Omega_{e}$, we have:

$\frac{\epsilon_{\Omega e}}{\epsilon_{\Omega e}^{*}}=\left(\frac{h_{e}}{h_{e}^{*}}\right)^{p+1}$.

where $\epsilon_{\Omega_{e}}^{*}$ is the relative error defined in the region enclosed by $\Omega_{e}$ in the optimal mesh. The total error in the optimal mesh is then:

$$
\epsilon^{* 2}=\sum_{e} \epsilon_{\Omega_{e}}^{* 2}=\sum_{e} \epsilon_{\Omega_{e}}^{2}\left(\frac{h_{e}^{*}}{h_{e}}\right)^{2(p+1)}=\sum_{e} \epsilon_{\Omega_{e}}^{2} r_{e}^{-2(p+1)},
$$

where $r_{e}$ is a factor that represents the reduction of element sizes in element $\Omega_{e}$. The number of elements $N^{*}$ in the optimal mesh can be computed using the $r_{e}$ 's. Clearly,

$N^{*}=\sum_{e} r_{e}^{d}$,

where $d$ is the dimension of the problem. Here $d=2$. The problem is either to minimize $N^{*}$ while controlling $\epsilon^{*}=\bar{\epsilon}$ or to minimize $\epsilon^{*}$ while controlling $N^{*}=\bar{N}$. The first 


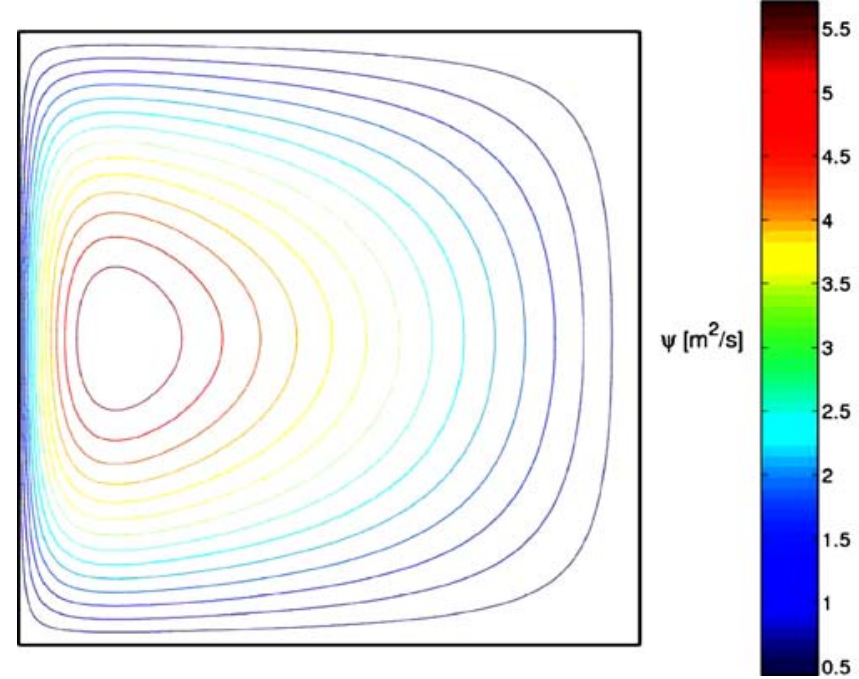

Fig. 5 Isolines of the stream function obtained for the Stommel model with the following parameters: $f_{0}=10^{-4} s^{-1}, \beta_{0}=$ $210^{-11} \mathrm{~m}^{-1} \mathrm{~s}^{-1}, \tau_{0}=10^{-1} \mathrm{Nm}^{-2}, \quad \gamma=10^{-6} \mathrm{~s}^{-1}, g=10 \mathrm{~ms}^{-2}$, $h=10^{3} \mathrm{~m}, \rho=10^{3} \mathrm{kgm}^{-3}$ and $L_{x}=L_{y}=10^{6} \mathrm{~m}$ the length of the domain along the $x$ and $y$ dimensions

problem leads to the following saddle point optimization problem:

$\left.\min _{r_{e}} \max _{\lambda} \sum_{e} r_{e}^{2}+\lambda \sum_{e} \epsilon_{\Omega_{e}}^{2} r_{e}^{-2(p+1)}-\bar{\epsilon}^{2}\right)$,

where $\lambda$ is a Lagrange multiplier. We find easily that:

$r_{e}^{2}=\left(\lambda(p+1) \epsilon_{\Omega_{e}}^{2}\right)^{1 /(p+2)}$.

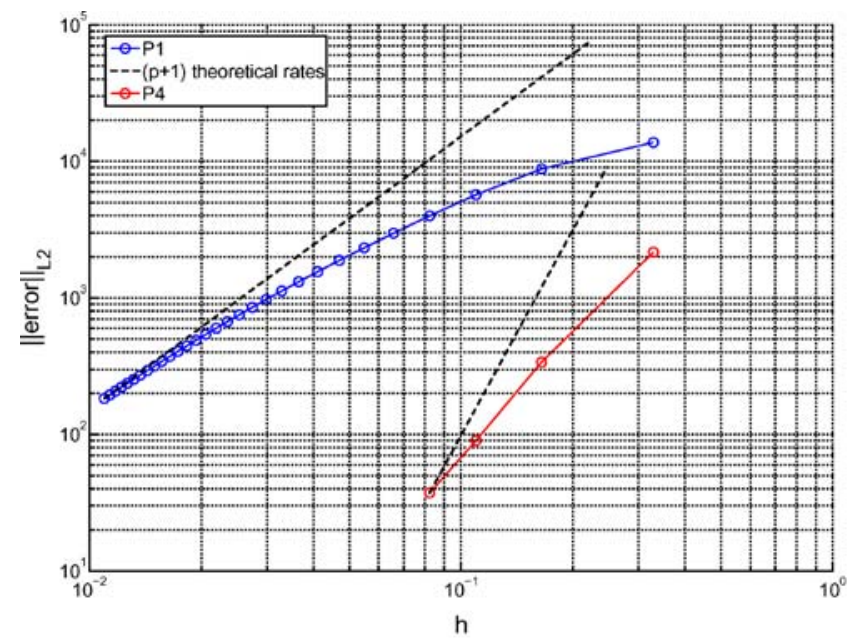

(a) structured mesh
The solution of the problem can be computed in a closed form if $p$ is constant:

$r_{e}=\overline{\boldsymbol{\epsilon}}^{1 /(p+1)} \boldsymbol{\epsilon}_{\Omega_{e}}^{-1 /(p+2)}\left[\sum_{e} \epsilon_{\Omega_{e}}^{2 /(p+2)}\right]^{-1 /(2 p+2)}$.

The second problem leads to:

$\left.\min _{r_{e}} \max _{\lambda} \sum_{e} \epsilon_{\Omega_{e}}^{2} r_{e}^{-2(p+1)}+\lambda \sum_{e} r_{e}^{2}-\bar{N}^{*}\right)$,

where $\lambda$ is another Lagrange multiplier. The solution is, for $p$ constant:

$r_{e}=\sqrt{N^{*}} \epsilon_{\Omega_{e}}^{1 /(p+2)}\left[\sum_{e} \epsilon_{\Omega_{e}}^{2 /(p+2)}\right]^{-1 / 2}$.

The $r_{e}$ 's define the size field used to build the adapted mesh by means of local mesh modifications.

\subsection{Projection of the solution}

Once the mesh has been adapted, the solution on the previous mesh is projected on the adapted one. This is done by means of an $L^{2}$ projection. The DG method allows this projection to be done element by element. Another advantage of the DG method is that, during the edge split operation, the projection is exact and no error is introduced. The edge collapse operator is only used in regions of the domain where the error is low, so no significative error is introduced with this coarsening operation. Finally, the

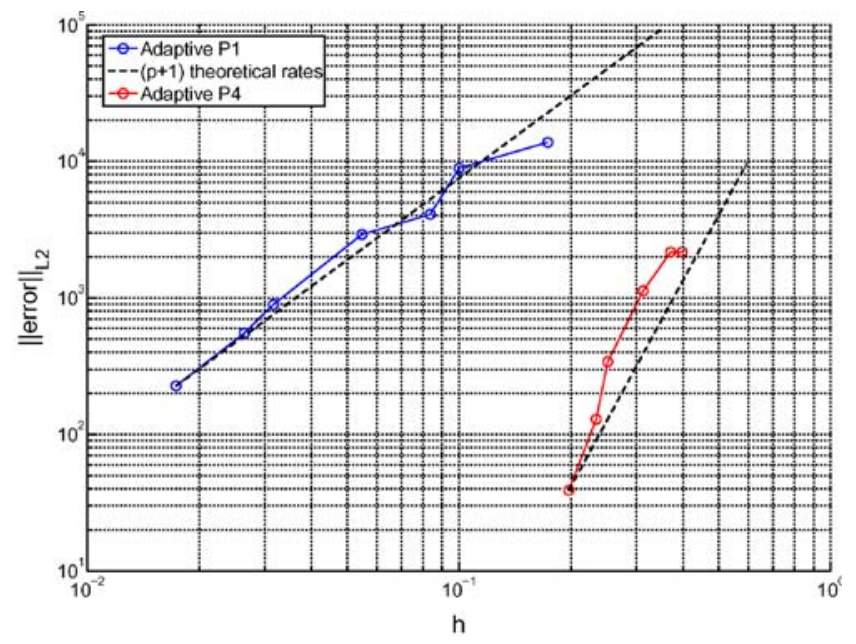

(b) adaptive mesh

Fig. 6 Convergence of the $L^{2}$ norm of the error $\epsilon$ vs the local mesh size $h$ using a uniformly refined meshes and using $\mathbf{b}$ adaptively refined meshes 
Fig. 7 The evolution of the maximum error, located in the western boundary layer for this Stommel model, showing the advantages of coupling both adaptivity and high order elements, which can be done in a simple and efficient way with the DG method

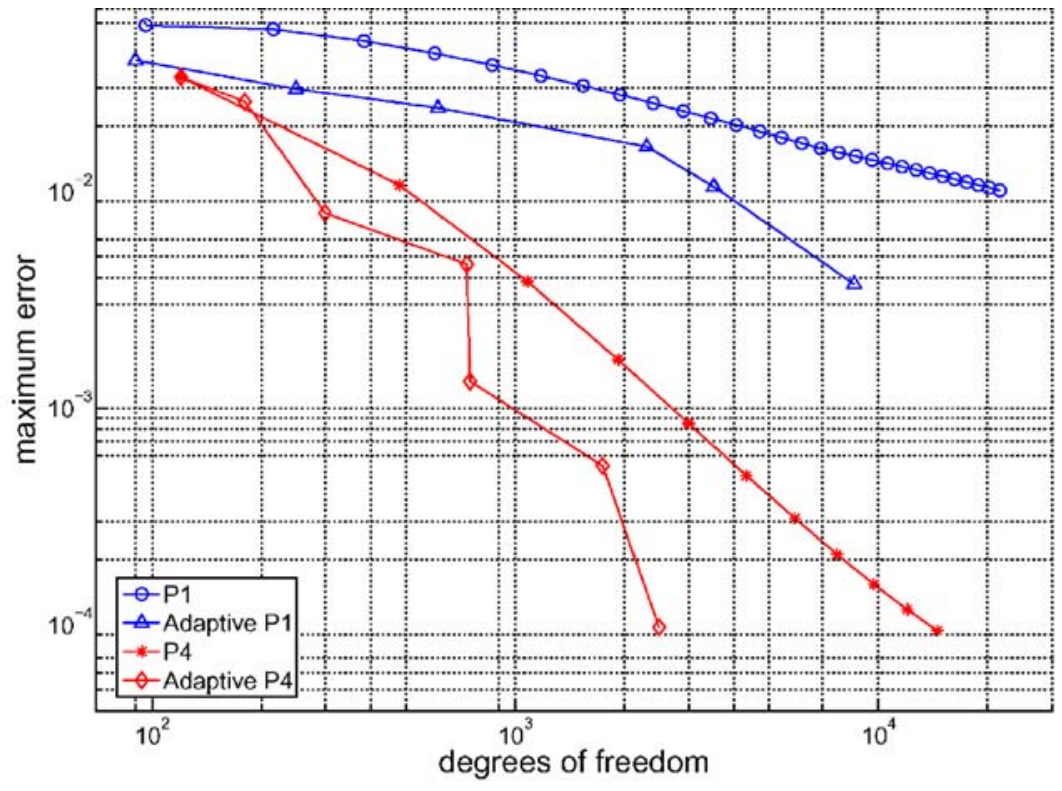

(a) Evolution of the maximum error vs. degrees of freedom

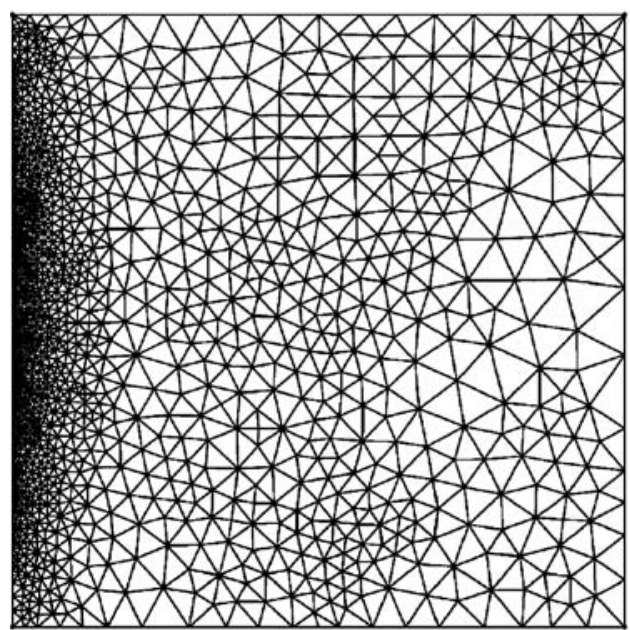

(b) 1 st order adaptive mesh

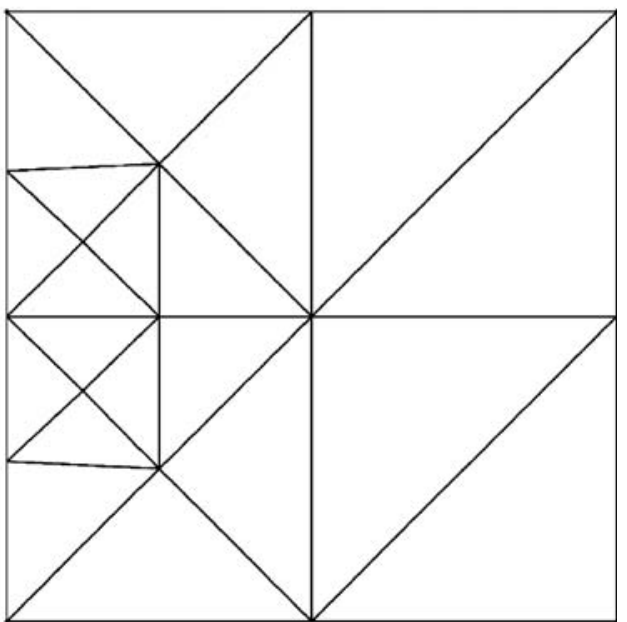

(c) 4th order adaptive mesh $e_{\max }=10^{-2}$ introduction of numerical diffusion is only expected when the swapping operation is applied. It is recommended to use an accurate integration scheme in the $L^{2}$ projection: the solution may be discontinuous across the edge that is swapped. Here, we do not consider node repositioning, typically Laplacian smoothing, because this mesh modification pattern introduces an excessive amount of numerical dissipation.

\section{Application to ocean modelling}

In this section, we first perform a validation step on a benchmark problem: the Stommel model. An adaptive convergence experiment is performed to test both the convergent behaviour of the DG scheme and the efficiency of the adaptive strategy. In a second experiment, we simulate the propagation of a typical anticyclonic baroclinic eddy in the realistic domain of the Gulf of Mexico.

\subsection{Adaptive convergence applied to the Stommel gyre}

Interesting simplifications can be done to the shallow water equations to obtain the Stommel model, used to perform the following convergence study.

First, the non linear transport terms $\nabla \cdot(H \mathbf{v v})$ are neglected. Then we assume a constant bathymetry and the $\beta$-plane approximation, according to which the Coriolis 


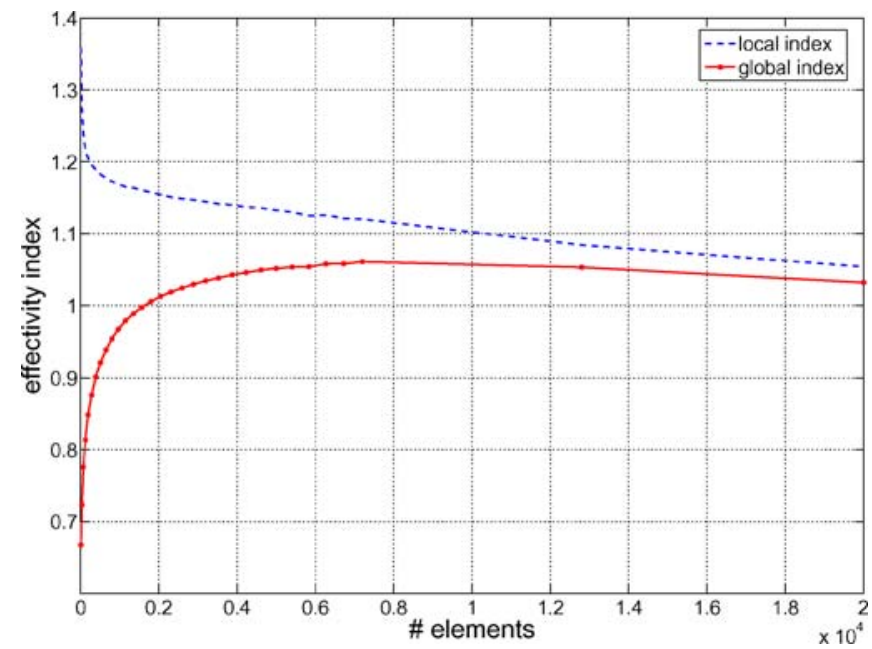

Fig. 8 The global and local effectivity index computed with first order elements both tend to one, indicating that the jumps can be considered as efficient error estimators

parameter is a linear function of one space coordinate, i.e. $f=f_{0}+\beta_{0} y$, where $f_{0} \approx 10^{-4} \mathrm{~s}^{-1}$ and $\beta_{0} \approx 10^{-11} \mathrm{~m}^{-1}$ $s^{-1}$ are constants. The dissipation term is parametrized as $\tau^{b}=\gamma H \mathbf{v}$ where $\gamma$ is a constant friction coefficient. The typical surface stress is given by $\tau^{s}=\tau_{0} \sin \left(\pi y^{\prime}\right)$ with $y^{\prime}=\frac{y}{L_{y}} \in[-0.5 ; 0.5]$ the non-dimensional coordinate with $L_{y}$ the typical size of the domain along the $y$-dimension. Finally, the relative elevation is neglected compared to the bathymetry, leading to the following classical linearization: $H=h+\eta \cong h$. With those approximations, the linearized form of the shallow water equations becomes:

$\frac{\partial \eta}{\partial t}+\nabla \cdot(h \mathbf{v})=0$

$$
\frac{\partial \mathbf{v}}{\partial t}+g \nabla \eta+\left(f_{0}+\beta_{0} y\right) \mathbf{e}_{z} \times \mathbf{v}=-\gamma \mathbf{v}+\frac{\tau^{s}}{h \rho}
$$

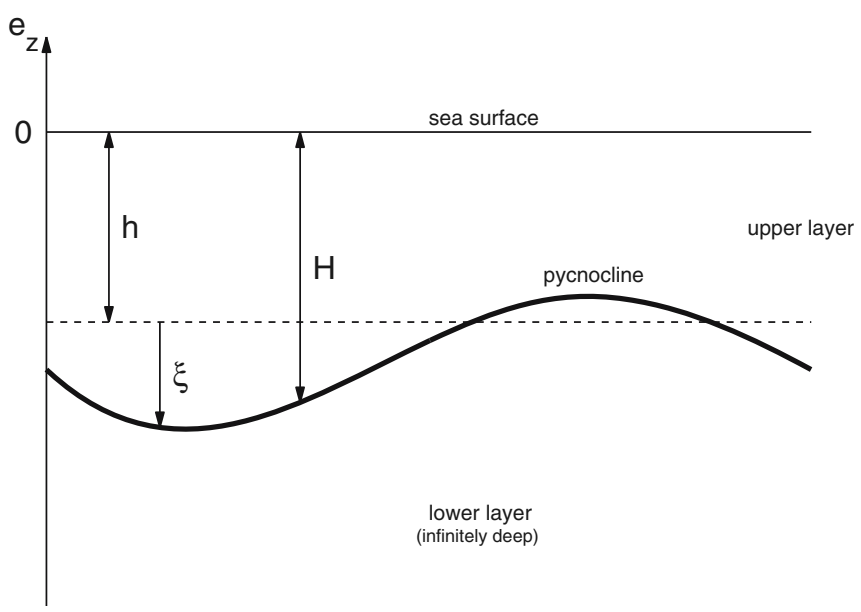

Fig. 10 Notations for the shallow water reduced gravity model, with the time-independent height $h$ and $\xi$ the downward displacement of the interface between the two layers

Equations 17 and 18 are sometimes called the Stommel model which leads to the typical "Stommel gyre" (Fig. 5; e.g. Stommel 1948).

The Stommel equations are solved on a square of $1,000 \mathrm{~km}$ of side, and compared to the analytical solution. The Coriolis effect leads to a geostrophic balance, creating a recirculation cell. The linear part $\beta y$ of the Coriolis factor $f$ tends to move the eddy westward (for the northern hemisphere parameters), leading to a boundary layer at the western boundary of the domain. The size of this boundary layer is determined by the ratio $\frac{\gamma}{\beta L_{x}}$. The adaptive method is therefore very useful to capture the large gradients on this western boundary, while the eastern part of the domain does not require such a fine discretization. The analytical solution can be found in Appendix A. On Fig. 5, the typical Stommel stream function is shown with its western boundary layer.
Fig. 9 The local effectivity fields present the same "boundary layer structure"

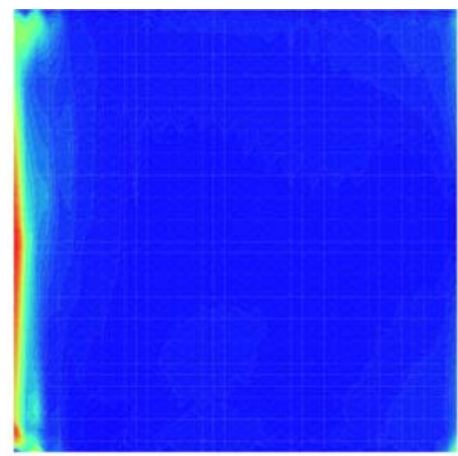

effectivity index

$2.141 e+00$ $4.535 e+00$

(a) 3200 elements structured grid
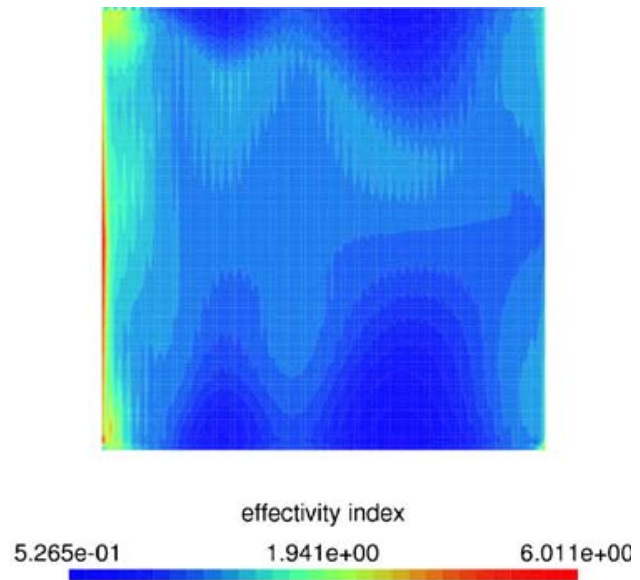

(b) 20000 elements structured grid 


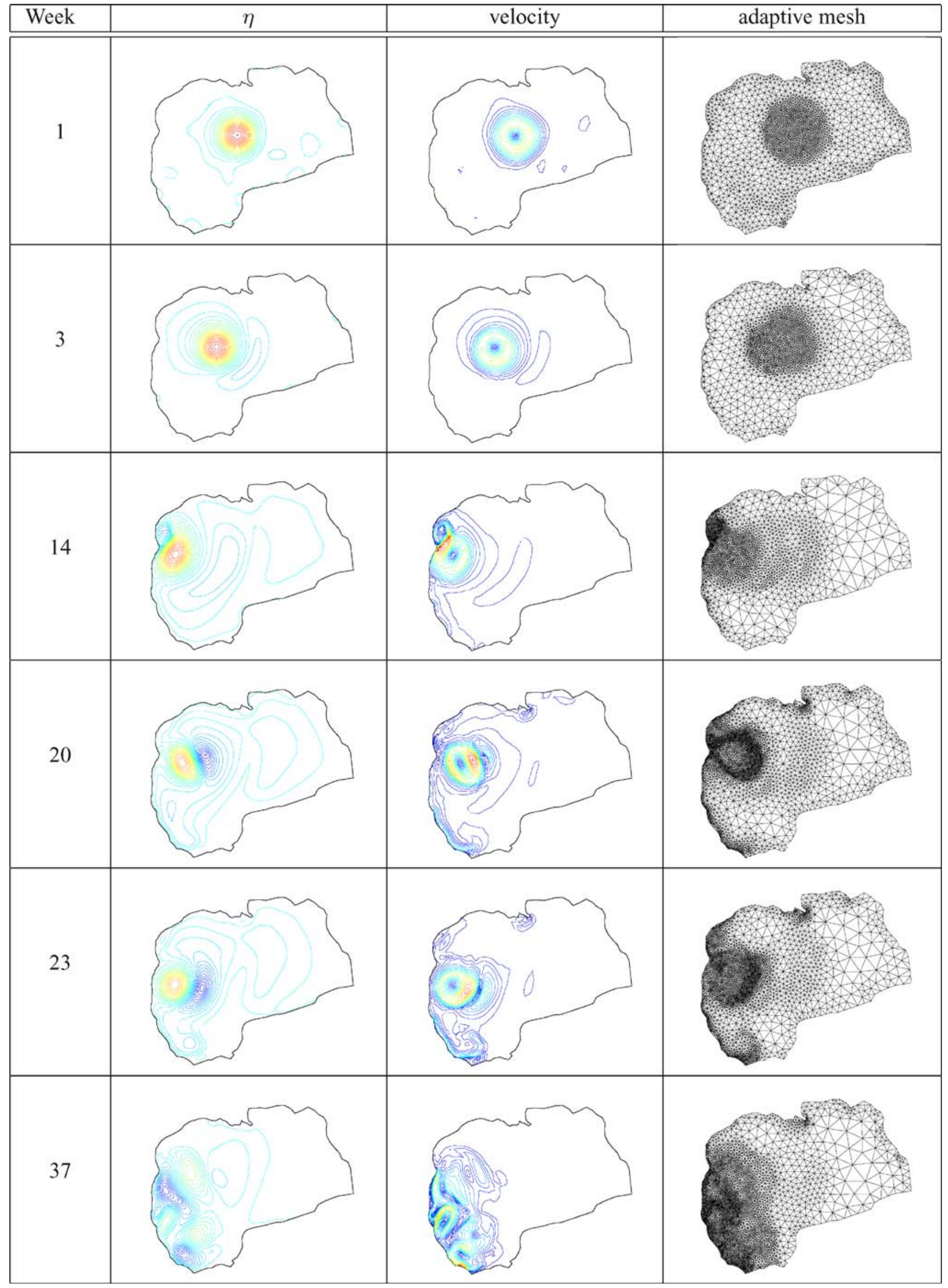


Fig. 11 Isolines of elevation field and velocity norm, and the corresponding adaptive mesh, respectively, at different times of the eddy propagation. On each plot, the 40 iso- $\eta$ lines extend from $-35 m$ (blue lines) to $65 m$ (red lines), while the 20 isovelocity lines extend from $0 \mathrm{~m} / \mathrm{s}$ to $1.5 \mathrm{~m} / \mathrm{s}$

Two different polynomial orders (P1 and P4 elements) have been used, giving two different theorical convergence rates, according to $h_{e}^{p+1}$.

Figure 6a presents the evolution of the $L^{2}$ error norm with the characteristic element size on uniformly refined meshes. The numerical results show that the theoretical asymptotic behaviour is only attained using dense uniform meshes.

The Fig. $6 \mathrm{~b}$ presents the evolution of the $L^{2}$ norm of the error versus the characteristic size of the elements corresponding to a given target error. The numerical results are compared to the theorical convergence rates lines.

Numerical results fit the theorical rates, both with fixed regular mesh and adapted mesh. A kind of oscillatory behaviour is still present on the adaptive mesh which can be explained with the use of the interval $[0.5 ; 1.4]$ to transform the error estimation into a new edge size field, as discussed in the previous paragraph. To reach the same error norm of approximately $5 \times 10^{-3}$ with linear shape functions, the non-adaptive structured regular mesh required 648 elements while the adaptive method needs only 79 elements. On the same mesh, the error is more than a hundred times smaller with $P 4$ element than with $P 1$.

For the same convergence experiment, we plot the maximum error compared to the degrees of freedom. As we see on Fig. $7 \mathrm{a}$, for the same maximum error of $10^{-2}$, the adaptive strategy with first order elements leads to use five times less degrees of freedom. And with the use of fourth order elements, we obtain about 100 less degrees of freedom for this same maximum error. The meshes on Figs. $7 \mathrm{~b}$ and $\mathrm{c}$ are, respectively, those obtained with the first and fourth order adaptive meshes.

Finally, a classical way to quantify the quality of an error estimator is the effectivity index (e.g. Ainsworth 2004), defined as the ratio between the norm of the error estimator $\epsilon$ and the norm of the exact error $e$. The global index is:

$\theta=\frac{\|\epsilon\|_{\mathcal{L}_{2}}}{\|e\|_{\mathcal{L}_{2}}}$

while the local one is defined in the same way on each element. With the first order elements, mean jumps have been defined on each node as the mean of the jumps on adjacent elements to compute the norm $\|\boldsymbol{\epsilon}\|_{L_{2}}$. Figure 8 shows the evolution of this index with the number of elements. The local index on this figure is defined as the simple mean of locals indexes on the whole domain. The global index tends to a value of 1.03 . A value so close to one indicates that the jumps seem to be relevant as error estimator. The local effectivity field is depicted on Fig. 9.
Our choice of mesh size field based on the only interface element jump leads to the right convergence rate, regardless of polynomial order. Adaptivity and the use of high order elements both seem useful, but combining the two seems particularly efficient and simple with the DG method.

\subsection{Propagation of a baroclinic anticyclonic eddy at midlatitudes}

The following results concern the propagation of a typical baroclinic anticyclonic eddy at midlatitudes (e.g. Hanert et al. 2005) in the Gulf of Mexico. The basin is assumed closed, the Yucatan Channel and the Florida Straits with their inflow and outflow are ignored. Although this experiment is highly idealized, it is expected to represent some of the features of the life cycle of anticyclonic eddies with the adaptive capture of eddies propagation.

To model the flow in the Gulf of Mexico, it is essential to take into account its baroclinic aspects. In this respect, the simplest approach consists in assuming that the domain is divided into two constant density layers separated by a free, impermeable interface: the pycnocline. As the lower layer is much deeper than the top layer, it is possible to further simplify the model to a reduced-gravity one. Such a model is widely used in oceanography and limnology (e.g. Naithani et al. 2003; Luthar and O'Brien 1985; Woodberry et al. 1989; Busalacchi and O'Brian 1980). To establish reduced-gravity equations, a closure hypothesis on the pressure force is needed, that eventually leads to a closed set of equations governing the motion in the surface layer. Accordingly, the downward displacement of the pycnocline, $\xi$, and the depth-averaged velocity in the surface layer, $v$, obey equations that are similar to the classical shallow-water equations introduced in section 2 :

$\frac{\partial \xi}{\partial t}+\nabla \cdot(H \mathbf{v})=0$

$\frac{\partial H \mathbf{v}}{\partial t}+\nabla \cdot(H \mathbf{v v})+f \mathbf{e}_{z} \times \mathbf{v}=-g^{\prime} H \nabla \xi$,

where $H=h+\xi$ is the actual depth of the upper layer (Fig. 10), given that $h$ is the unperturbed equilibrium height of the surface layer; $g^{\prime}$ is the so-called reduced gravity. The latter is defined as $g^{\prime}=g \delta$, with $g$ the gravity and $\delta=\frac{\Delta \rho}{\rho}$ the relative density difference between the bottom and the surface layers.

A Gaussian distribution of water elevation $\eta$ is assumed at initial time:

$\eta(x, y, t=0)=C \exp \left[-D\left(x^{2}+y^{2}\right)\right]$,

with $C=68.2 \mathrm{~m}$ and $D=5.92 \times 10^{-11} \mathrm{~m}^{-2}$. The $\beta$-plane assumption is made (i.e. $f=f_{0}+\beta y$ ) with the Coriolis 
parameters taken at $25 N: f_{0} \cong 6.163510^{-5} s^{-1}$ and $\beta_{0} \cong$ $2.074610^{-11} \mathrm{~m}^{-1} \mathrm{~s}^{-1}$. An initial velocity field is taken to be in geostrophic balance, which means:

$$
u(\mathbf{x}, t=0)=-\frac{g}{f} \frac{\partial \eta}{\partial y}=2 \frac{g^{\prime}}{f_{0}+\beta_{0} y} C D y \exp \left[-D\left(x^{2}+y^{2}\right)\right],
$$$$
v(\mathbf{x}, t=0)=\frac{g}{f} \frac{\partial \eta}{\partial x}=-2 \frac{g^{\prime}}{f_{0}+\beta_{0} y} C D x \exp \left[-D\left(x^{2}+y^{2}\right)\right],
$$

leading to a maximum initial flow speed of $1 \mathrm{~ms}^{-1}$ with $g^{\prime}=0.137 \mathrm{~ms}^{-2}$ and $h=100 \mathrm{~m}$. The maximum Froude number reached during the simulation is $\max (F r)=0.5$, which is very high compared to the simple Stommel model, and the Rossby number is $R o \cong 9 \times 10^{-3}$.

No wind forcing and no bottom friction is applied. The Coriolis effect is thus the only source term responsible for moving the eddy westward. This propagation of slow Rossby waves, represented with the shallow water equations, is due to the $\beta$-effect. Such waves have a major effect on the large scale circulation, and thus on weather and climate. For instance, Rossby waves can intensify western boundary currents, which transport huge quantities of heat. Even a minor shift in the position of the current can thus affect weather over large areas of the globe.

Figure 11 represents the evolution of the eddy with the Coriolis forcing. Its westward propagation is captured by the mesh evolution (right column): a minimum size field of $10 \mathrm{~km}$ has been applied to keep a low number of elements.

After 1 week of physical time simulation, the mesh has been adapted three times to capture the regions with larger variations: the eddy region, and the coast lines, where gravity waves come and bounce back. At this time, the mesh presents approximately 3,000 elements. The eddy keeps moving westward until approximately week 11, when it reaches the coast. Then, its shape is modified when a second eddy appears, spinning in the opposite direction. The number of elements grows up to 7,000 to fit this large variation region. On week 14 , one can see the slow creation of a western boundary current, flowing southward. As the eddy keeps moving southward, the mesh seems to perfectly capture the evolution of this current and the eddy generated at the southern extremity of the golf on week 23 . The initial eddy then collapses to generate smaller eddies which keep spinning and mixing on the western boundary. The number of elements decreases then to the initial value of approximately 3,000 .

The mesh has been well adapted to eddies and currents, but large field variations and mesh refinement must also be noticed on sharp and non regular coasts, as on the north and east-north of the Gulf. A restriction of $10 \mathrm{~km}$ has been applied on the size of elements on adaptive mesh. To reach the same size with a non-adaptive mesh, about 34,000 elements would be required with the first order polynomial shape functions.

\section{Conclusion}

In this paper, we show that both high-order elements and mesh adaptivity, coupled with discontinuous Galerkin method, can be a very attractive approach to simulate ocean flows. The DG method provides a simple and efficient way to deal with those two techniques, by providing an efficient error estimator. This DG method has been successfully applied to the shallow water equations, and seems to be particularly promising in this framework of a new ocean model. Of course, in terms of the physic that need to be modelled, lots of work need to be done in code implementation to compare with established ocean model. Our next step towards this goal will be the ability to deal with realistic bathymetry. On the mesh adaptation side of the work, we plan to take full advantage of the anisotropic mesh adaptation features of the MeshAdapt package.

Acknowledgment Eric Deleersnijder is a Research associate with the Belgian National Fund for Scientific Research. The present study was carried out within the scope of the project "A second-generation model of the ocean system", which is funded by the Communauté Française de Belgique, as Actions de Recherche Concertées, under contract ARC 04/09-316. This work is a contribution to the SLIM ${ }^{2}$ project. The authors gratefully acknowledge Emmanuel Hanert for the comments and help he provided during the preparation of this paper.

\section{Appendix}

1.1 Analytical solution of the Stommel problem

The analytical steady solution is given by:

$$
\begin{aligned}
& \Psi(x, y)=\frac{D^{3} \tau_{0} L_{y}}{\pi^{2} \gamma \rho} f_{1}(x) \cos (\pi y) \\
& U(x, y)=\frac{D \tau_{0}}{\pi \gamma \rho} f_{1}(x) \sin (\pi y) \\
& V(x, y)=\frac{D \tau_{0}}{\pi \gamma \rho \delta} f_{2}(x) \cos (\pi y) \\
& \eta(x, y)=\frac{D \tau_{0} f_{0} L_{x}}{\pi \gamma \rho \delta g h}\left[-\frac{C_{d r a g}}{\delta \pi} f_{2}(x) \sin (\pi y)\right. \\
& \left.\quad+\frac{1}{\pi} f_{1}(x)\left(\cos (\pi y)(1+\beta y)-\frac{\beta}{\pi} \sin (\pi y)\right)\right]
\end{aligned}
$$

with the following functions:

\footnotetext{
${ }^{2}$ SLIM, Second-Generation Louvain-la-Neuve Ice-ocean Model, www.climate.be/SLIM
} 


$$
\begin{aligned}
f_{1}(x) & =\frac{\pi}{D}\left(1+\frac{\left(e^{z-}-1\right) e^{z+x}+\left(1-e^{z+}\right) e^{z-x}}{e^{z}+-e^{z-}}\right) \\
f_{2}(x) & =\frac{1}{D} \frac{\left(e^{z-}-1\right) z+e^{z+x}+\left(1-e^{z+}\right) z-e^{z-x}}{e^{z+}-e^{z-}} \\
D & =\frac{\left(e^{z-}-1\right) z_{+}+\left(1-e^{z+}\right) z_{-}}{e^{z+}-e^{z-}} \\
z_{-}^{+} & =\frac{-1 \pm \sqrt{1+(2 \pi \delta \epsilon)^{2}}}{2 \epsilon}
\end{aligned}
$$

The dimensionless parameters used are: the aspect ratio of the domain $\delta=\frac{L_{x}}{L_{y}}$, the ration between bottom friction and Coriolis effect $C_{d r a g}=\frac{\gamma}{f_{0}}$ and $\epsilon=\frac{\gamma}{L_{x} \beta_{0}}$ the parameter defining the boundary layer width.

\section{References}

Adjerid S, Devine KD, Flaherty JE, Krivodonova L (2002) A posteriori error estimation for discontinuous Galerkin solutions of hyperbolic problems. Comput Methods Appl Mech Eng 191:1097-1112

Ainsworth M (2004) Dispersive and dissipative behavior of high order discontinuous Galerkin finite element methods. J Comput Phys 198(1):106-130

Atkins HL, Shu C-W (1998) Quadrature-free implementation of discontinuous Galerkin methods for hyperbolic equations. AIAA J 36(5):775-782

Baker TJ (1997) Mesh adaptation strategies for problems in fluid dynamics. Finite Elem Anal Des 25(3-4):243-273

Bassi F, Rebay S (1997) A high-order accurate discontinuous finite element solution of the $2 \mathrm{~d}$ Euler equations. J Comput Phys 138:251-285

Behrens J (1998) Atmospheric and ocean modeling with an adaptive finite element solver for the shallow-water equations. Appl Numer Math 26:217-226

Busalacchi AJ, O'Brian JJ (1980) The seasonal variability in a model of the tropic pacific. J Phys Oceanogr 10:1929-1951

Chevaugeon N, Hillewaert K, Gallez X, Ploumhans P, Remacle J-F (2005a) Optimal numerical parameterization of discontinuous Galerkin method applied to wave propagation problems. J Comput Phys (in press)

Chevaugeon N, Remacle J-F, Gallez X, Ploumans P, Caro S (2005b) Efficient discontinuous Galerkin methods for solving acoustic problems. In: 11th AIAA/CEAS Aeroacoustics Conference

Chevaugeon N, Xin J, Hu P, Li X, Cler D, Flahertyand JE, Shephard MS (2005c) Discontinuous Galerkin methods applied to shock and blast problems. J Sci Comput 22(1):227-243

Cockburn B, Karniadakis GE, Shu C-W (2000) Discontinuous Galerkin methods. Lect Notes Comput Sci Eng. Springer, Berlin Heidelberg New York

Dawson C, Proft J (2002) Discontinuous and coupled continuous/ discontinuous Galerkin methods for the shallow water equations. Comput Methods Appl Mech Eng 191(4142):4721-4746

Dawson C, Proft J (2004) Coupled discontinuous and continuous Galerkin finite element methods for the depth-integrated shallow water equations. Comput Methods Appl Mech Eng 193(3-5)

George PL, Borouchaki H, Laug P (2002) An efficient algorithm for 3d adaptive meshing. Adv Eng Softw 33(7-10):377-387, ISSN 09659978. DOI 10.1016/S0965-9978(02)00065-0
Giraldo FX, Hesthaven JS, Warburton T (2002) Nodal high-order discontinuous Galerkin methods for the spherical shallow water equations. J Comput Phys 181:499-525

Hanert E, Le Roux DY, Legat V, Deleersnijder E (2004) Advection schemes for unstructured grid ocean modelling. Ocean Model 7:39-58

Hanert E, Le Roux DY, Legat V, Deleersnijder E (2005) An efficient finite element method for the shallow water equations. Ocean Model 10:115-136

Heinze T, Hense A (2002) The shallow water equations on the sphere and their lagrange-Galerkin-solution. Meteorol Atmos Phys 81:129-137

Li X (2003) Mesh modification procedure for general 3-D nonmanifold domains. $\mathrm{PhD}$ thesis, Renselear Polytechnic Institute

Li X, Remacle J-F, Chevaugeon N, Shephard MS (2004) Anisotropic mesh gradation control. In 13th International Meshing Roundtable

Lockard DP, Atkins HL (1999) Efficient implementations of the quadrature-free discontinuous Galerkin method. In Proceeding of 14th AIAA CFD conference. AIAA

Luthar ME, O'Brien JJ (1985) A model of the seasonal circulation in the Arabian sea forced by observed winds. Progr Oceanogr 14:353-385

Marchandise E, Chevaugeon N, Remacle J-F (2005) Spatial and spectral superconvergence of discontinuous Galerkin methods applied to hyperbolic problems. J Comput Appl Math, accepted for publication

Nair RD, Thomas SJ, Loft RD (2005) A discontinuous Galerkin global shallow water model. Mon Weather Rev 133:876-888

Naithani J, Deleersnijder E, Plisnier P-D (2003) Analysis of windinduced thermocline oscillations of lake tanganyika. Environ Fluid Mech 3:23-39

Pain CC, Piggott MD, Goddard AJH, Fang F, Gorman GJ, Marshall DP, Eaton MD, Power PW, de Oliveira CRE (2005) Threedimensional unstructured mesh ocean modelling. Ocean Model 10:5-33

Pietrzak J, Deleersnijder E, Schroeter J (eds) (2005) The second international workshop on unstructured mesh numerical modelling of coastal, shelf and ocean flows (delft, the Netherlands, September 23-25, 2003). Ocean Model (special issue) $10: 1-252$

Remacle J-F, Li X, Shephard MS, Flaherty JE (2005) Anisotropic adaptive simulation of transient flows using discontinuous Galerkin methods. Int J Numer Methods Eng 62(7):899-923

Remacle J-F, Soares Frazão S, Li X, Shephard MS (2006) An adaptive discretization of shallow-water equations based on discontinuous Galerkin methods. Int J Numer Methods Fluids 52:903-923

Roe PL (1981) Approximate Riemann solvers, parameter vectors and difference schemes. J Comput Phys 43:357-372

Schwanenberg D, Kongeter J (2000) A discontinuous Galerkin method for the shallow water equations with source terms. Comput Sci Eng 11:419-424

Soares Frazão S, Zech Y (2002a) Undular bores and secondary waves-experiments and hybrid finite-volume modelling. J Hydraulic Res 40:33-34

Soares Frazão S, Zech Y (2002b) Dam-break in channels with 90degree bend. J Hydraul Res, American Society of Civil Engineers 128:956-968

Speares W, Berzins M (1997) A 3d unstructured mesh adaptation algorithm for the time-dependent shock-dominated problems. Int J Numer Methods Fluids 25(1):81-104

Stommel H (1948) The westward intensification of wind-driven ocean currents. Transactions-American Geophysical Union 29:202-206

Vreugdenhill CB (1994) Numerical methods for shallow water flow. Water Science and Technologies Library. Kluwer

Woodberry KE, Luther ME, O'Brien JJ (1989) The wind-driven seasonal circulation in the southern tropical Indian ocean. J Geophys Res 94:17,985-18,002 


\section{Erratum}

This erratum concerns the appendix of the paper Highorder h-adaptive discontinuous Galerkin methods for ocean modeling, Ocean Dynamics, 57, 109-121.

Although presented only for completeness and not being used in our computations the analytical stream function $\Psi$ of the Stommel gyre test case given in the appendix on page 120 is wrong. The non dimensional function $\mathcal{D}^{3}$ should read $\mathcal{D}$ while the velocity and water elevation remain unchanged. The analytical Stommel gyre solution then reads :

$$
\begin{aligned}
\Psi(x, y) & =\frac{\mathcal{D} \tau_{0} L_{y}}{\pi^{2} \gamma \rho} f_{1}(x) \cos (\pi y) \\
U(x, y) & =\frac{\mathcal{D} \tau_{0}}{\pi \gamma \rho} f_{1}(x) \sin (\pi y) \\
V(x, y) & =\frac{\mathcal{D} \tau_{0}}{\pi \gamma \rho \delta} f_{2}(x) \cos (\pi y) \\
\eta(x, y) & =\frac{\mathcal{D} \tau_{0} f_{0} L_{x}}{\pi \gamma \rho \delta g h}\left[-\frac{\gamma}{f_{0} \delta \pi} f_{2}(x) \sin (\pi y)\right. \\
& +\frac{1}{\pi} f_{1}(x)(\cos (\pi y)(1+\beta y) \\
& \left.\left.-\frac{\beta}{\pi} \sin (\pi y)\right)\right]
\end{aligned}
$$

with the following functions:

$$
\begin{aligned}
f_{1}(x) & =\frac{\pi}{\mathcal{D}}\left(1+\frac{\left(e^{z_{2}}-1\right) e^{x z_{1}}+\left(1-e^{z_{1}}\right) e^{x z_{2}}}{e^{z_{1}}-e^{z_{2}}}\right) \\
f_{2}(x) & =\frac{1}{\mathcal{D}} \frac{\left(e^{z_{2}}-1\right) z_{1} e^{x z_{1}}+\left(1-e^{z_{1}}\right) z_{2} e^{x z_{2}}}{e^{z_{1}}-e^{z_{2}}} \\
\mathcal{D} & =\frac{\left(e^{z_{2}}-1\right) z_{1}+\left(1-e^{z_{1}}\right) z_{2}}{e^{z_{1}}-e^{z_{2}}} \\
z_{2}^{1} & =\frac{-1 \pm \sqrt{1+(2 \pi \delta \epsilon)^{2}}}{2 \epsilon}
\end{aligned}
$$

and with $x$ and $y$ the non dimensional axis $x \in\left[\begin{array}{ll}0 & 1\end{array}\right]$ and $y \in[-0.50 .5]$. The dimensionless parameters used are

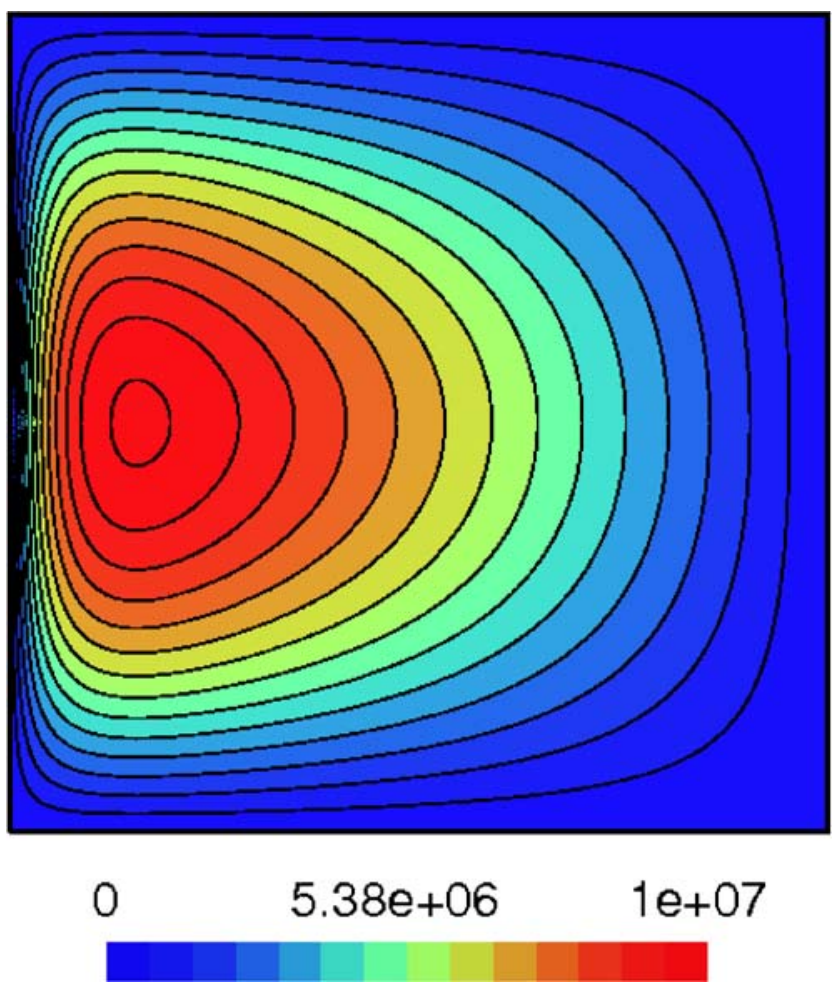

Fig. 1 Isolines of the stream function $\Psi(x, y)$ obtained for the Stommel model. Notice that $\Psi$ is the stream function of the transport so the units read $m^{3} s^{-1}$. The following standard parameters are used: $f_{0}=10^{-4} \mathrm{~s}^{-1}, \beta=210^{-11} \mathrm{~m}^{-1} \mathrm{~s}^{-1}, \tau_{0}=10^{-1} \mathrm{Nm}^{-2}$, $\gamma=10^{-6} \mathrm{~s}^{-1}, g=10 \mathrm{~ms}^{-2}, \mathrm{~h}=10^{3} \mathrm{~m}, \rho=10^{3} \mathrm{kgm}^{-3}$ and $L_{x}=L_{y}=10^{6} \mathrm{~m}$ the length of the domain along the $x$ and $y$ dimensions

the aspect ratio of the domain $\delta=\frac{L_{x}}{L_{y}}$ and $\epsilon=\frac{\gamma}{L_{x} \beta}$ the parameter defining the boundary layer width.

This correction on the stream function expression requires a modification of figure 5 on page 115 . The corrected function is depicted in Fig. 1.

We would like to thank the students of J.-O. Wolff and K. Lettmann at the University of Oldenburg who found this error during an exercise in their class on theoretical oceanography. 